\title{
Percepción de la confiabilidad de un producto agroindustrial
}

\author{
Goirán, Andrés Roque
}

PhD candidate at Universitat Politècnica de València, Spain. angoiran@hotmail.com.

\begin{abstract}
Resumen
Las máquinas que trabajan en la agricultura y sus partes componentes están predestinadas a cumplir las funciones asignadas en determinadas condiciones de producción y explotación técnica. El estado técnico de las maquinas durante el proceso de explotación cambia, asi como cambian de nominal al límite los valores de los parámetros que lo caracterizan.

Una forma de caracterizar la maquinaria agrícola que se produce, es aplicando el concepto de sistemas, el cual según la cantidad de sistemas que la conforman y el grado de nivel tecnológico de los mismos podemos definir: Productos de alta tecnología: Tractores, Cosechadoras; Productos de media Tecnología: Sembradoras, embutidora de granos, extractora de granos; Productos de baja tecnología: Implementos observándose una estrecha relación entre la confiabilidad y la caracterización tecnológica. Productos de alta tecnología son más confiables que productos media y baja por razones múltiples entre ellas gestión del diseño, procesos de fabricación, acceso a tecnología del conocimiento, nivel de facturación de la empresa origen, organización empresarial, valor del producto, competencia.

El interés de este trabajo es profundizar el concepto mecánico de confiabilidad, investigando como tal la percepción de la misma en el usuario, como concepto globalizador donde estará implícito el concepto mecánico; abordándose la problemática desde dos puntos de vista: la confiabilidad como factor de compra y la confiabilidad en función de la operatividad de la máquina.
\end{abstract}

Palabras clave: confiabilidad, caracterización, productos agroindustriales, usuario, gestión de diseño.

\footnotetext{
Abstract

Machines working in agriculture and its component parts are predestined to fulfill the functions assigned under certain conditions of production and technical operation. The technical condition of the machine during operation changes, and change the limit nominal values of the parameters that characterize it.

One way to characterize agricultural machinery produced, is applying the concept of systems, which according to the number of systems that conform and the degree of technological level of the same can define: High-tech products: tractors, harvesters;
} 
Medium-technology products: Drills, stocking stuffer grains, grain extractor; Low-tech products: simple machines observed a close relationship between reliability and technological characterization. High-tech products are more reliable than medium and low products for multiple reasons including design management, manufacturing processes, access to knowledge technology, level of turnover of the company source, business organization, product value, competition.

The interest of this work is to deepen the mechanical concept of reliability, investigating how such perception of it in the user, as globalization concept where the mechanical concept is implicit; the problem from two perspectives: reliability as purchasing factor and reliability depending on the operation of the machine.

Keywords: reliability, characterization, agro-industrial products, user, design management

\section{Introducción}

Uno de los factores determinantes en el éxito de un producto agrícola es el grado de confiabilidad del mismo, otros tienen que ver con la performance, precio de adquisición y reventa. En su conjunto estos factores forman el intangible de la Calidad del producto. (Bragachini, 2010).

En términos estadísticos la confiabilidad se define como la probabilidad de que un producto, parte de un equipo o sistema, lleve a cabo su función esperada en un período establecido de tiempo bajo condiciones especificadas de funcionamiento es decir que la confiablidad de un producto es una noción dinámica a través del tiempo. Desde el punto de vista puramente económico es deseable una alta fiabilidad para reducir los costos totales, ya que es inquietante el hecho de que el costo anual para mantener ciertos equipos y sistemas de funcionamiento ha llegado a ser varias veces mayor al costo original del equipo. (Shkiliova et al., 2007).

Las máquinas que trabajan en la agricultura y sus partes componentes están predestinadas a cumplir las funciones asignadas en determinadas condiciones de producción y explotación técnica. El estado técnico de las maquinas durante el proceso de explotación cambia, así como cambian de nominal al límite los valores de los parámetros que lo caracterizan (Shiliova et al., 2011). Cuando al menos un parámetro estructural sobrepasa su valor límite, esto puede provocar un deterioro o la pérdida de la capacidad del trabajo de la máquina o sus partes componentes. Las partes componentes de las máquinas se regulan, recuperan y sustituyen para lograr que sus parámetros técnicos no sobrepasasen durante el proceso de explotación el valor límite y los agregados no alcancen su estado límite. Precisamente es por esto que se utilizan en la documentación técnica normativa el sistema de los valores permisibles de los parámetros, que permitan prevenir las fallas y/o las consecuencias de las fallas de las piezas en el proceso de explotación hasta llegar a la próxima reparación o control de su estado técnico. Las maquinas modernas agrícolas (tractores, combinadas y otras) están constituidas por decenas de miles de piezas, cada cual potencialmente puede fallar por uno o varios parámetros de su estado técnico. Sin embargo, es conocido en la práctica de explotación que solamente hasta un 5\% de las piezas representan la fuente principal de las fallas. Debido a esto es que en la práctica durante el proceso de dirección del estado técnico y de confiabilidad de las máquinas, generalmente, se trabaja con 100-200 piezas, que condicionan el nivel de trabajo sin falla, durabilidad y con un nivel bajo de mantenimiento de las máquinas agrícolas (Shkiliova et al., 2011). 
Es decir que desde un punto de vista mecánico los componentes principales de una máquina agrícola son aquellos que determinan el funcionamiento en su conjunto, estos componentes forman parte de sistemas individuales no redundantes cuya configuración se asimila a un sistema en paralelo (J Shigley,2004) por lo que la confiabilidad será igual al producto de las confiabilidades individuales de los sistemas que lo integran.

Una forma de caracterizar la maquinaria agrícola que se produce, es aplicando el concepto de sistemas, el cual según la cantidad de sistemas que la conforman y el grado de nivel tecnológico de los mismos podemos establecer del siguiente modo: Productos de alta tecnología: Tractores, Cosechadoras; Productos de media Tecnología: Sembradoras, embutidora de granos, extractora de granos; Productos de baja tecnología: Implementos, observándose una estrecha relación entre la confiabilidad y la caracterización tecnológica. Productos de alta tecnología son más confiables que productos media y baja por razones múltiples entre ellas gestión del diseño, procesos de fabricación, acceso a tecnología del conocimiento, nivel de facturación de la empresa origen, organización empresarial, valor del producto, competencia.

\section{Objetivos e Hipótesis}

El interés de este trabajo es profundizar el concepto mecánico de confiabilidad, investigando como tal la percepción de la misma en el usuario, como concepto globalizador donde estará implícito el concepto mecánico; abordándose la problemática desde dos puntos de vista: la confiabilidad como factor de compra y la confiabilidad en función de la operatividad de la máquina.

Como objetivo se planteó determinar cuáles son las variables principales que influyen en cada caso, considerando como hipótesis que el servicio técnico prestado por la empresa fabricante funciona como una variable de equilibrio, lo que explicaría el fuerte arraigo territorial de las empresas agroindustriales.

\section{Descripción del área de estudio.}

La organización empresarial de las mayoría de las industrias agroindustriales de la Región Centro de la República Argentina tienen una base familiar concentrada en " manchones territoriales " influenciadas directamente por el peso de la agricultura y de la ganadería, tratándose de un mercado complejo y heterogéneo según la particularidad de la zona. Este conjunto de empresas tiene un denominador común: una extensa localización territorial acorde con las demandas productivas regionales. Sobre este total, la gran mayoría son básicamente pymes, con un promedio de 30 años de antigüedad, cuyo tamaño va desde 10 hasta 300 empleados por empresa (hay algunas pocas excepciones que superan ese límite). El resto del mercado se distribuye en los segmentos de mayor valor económico y complejidad tecnológica. 


\subsection{Distribución porcentual de máquinas de media y baja tecnología fabricadas en la Región Centro}

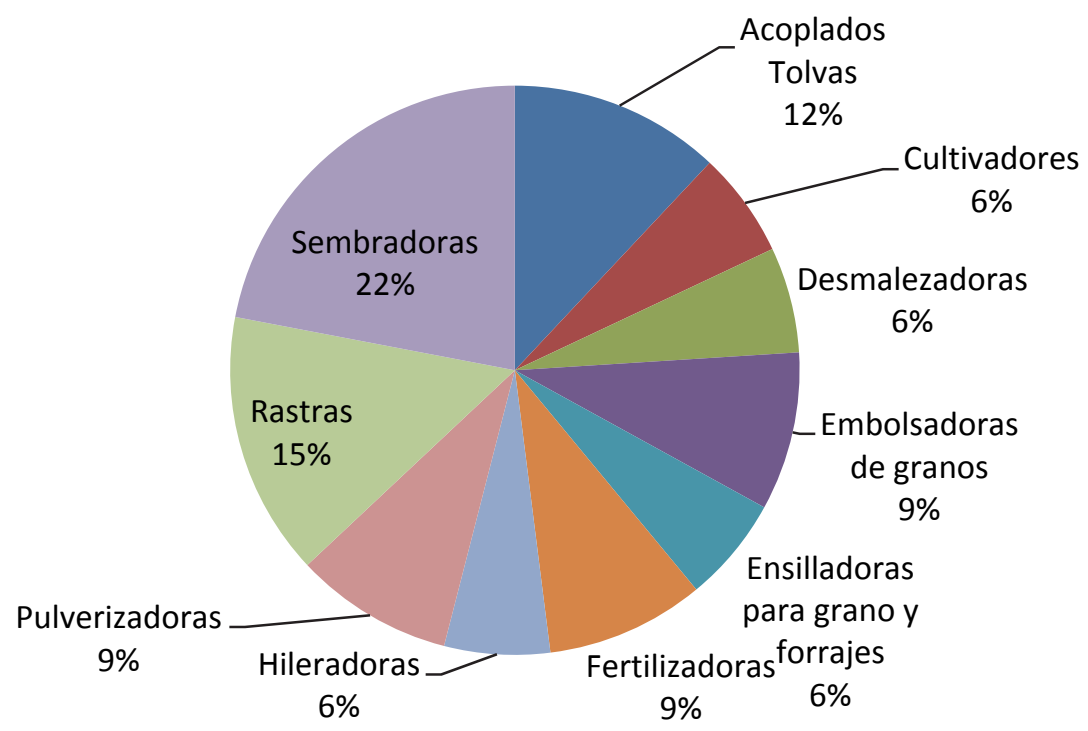

Fig. 1 Distribución porcentual de máquinas de media y baja tecnología fabricadas en la Región Centro.(fuente propia)

\section{Metodología}

Para analizar la confiabilidad como factor de compra, se realizó una encuesta en la cual se propusieron como variables de evaluación: Referencia por otro usuario, marca conocida en la zona, conocimiento del vendedor (relación personal), conocimiento de la fábrica (establecimiento donde se produce el producto agroindustrial), económico.En el análisis de la confiabilidad según la operación de la máquina, se realizó una encuesta en la cual se propusieron como variables de evaluación: Referencia por otro usuario, marca conocida en la zona, conocimiento del vendedor (relación personal), conocimiento de la fábrica (establecimiento donde se produce el producto agroindustrial), valor económico. La variable servicio técnico se analizó estratificando las encuestas desagregando las explotaciones agropecuarias según el siguiente criterio: productores de menos 200 ha, entre 200 y 600 ha, de entre 600 y 1500 ha y de más de 1500 ha. Las encuesta consto de un cuestionario de 15 preguntas de las cuales 10 fueron cerradas cuantificables y el resto abiertas, se llevaron a cabo durante la exposición agroindustrial "Agroactiva", en Mayo del 2014 en la ciudad de Oncativo provincia de Córdoba República Argentina. Los objetivos de la encuesta fueron: Definir y cuantificar las variables que involucran la percepción de confiabilidad en el cliente y establecer el rol del servicio técnico en la percepción de la confiabilidad.

\section{Muestreo}

El total de productores y contratistas entrevistados asciende a 600 con un nivel de confianza del $95,5 \%$, el universo por lo tanto lo constituye todos los productores y/o contratistas propietarios de por lo menos un producto agroindustrial de media o baja tecnología. 


\section{Resultados}

\subsection{Percepción de la confiabilidad del producto según el entorno}

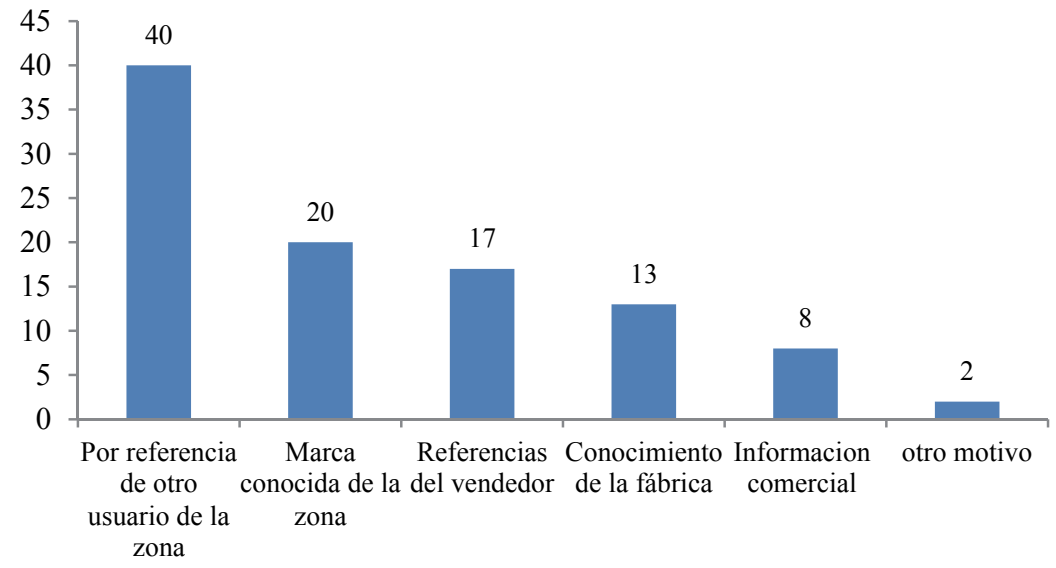

Fig. 2: Porcentaje de opiniones afirmativas respecto a las variables de confiabilidad del producto de acuerdo al entorno.

Según los resultados (Fig. 2) y considerando que la confiabilidad es un factor importante a la hora de adquirir un producto agroindustrial las variables "referencia de otro usuario " y " marca conocida" son las que más influirán más en el momento de la decisión de compra. Ambas suman el $60 \%$ del total. En tercer lugar se ubica la variable "referencias del vendedor" con el $17 \%$, indicando que la reputación técnica comercial es importante en la percepción.

\subsection{Valoración del servicio técnico como variable influyente en la percepción de la confiabilidad}

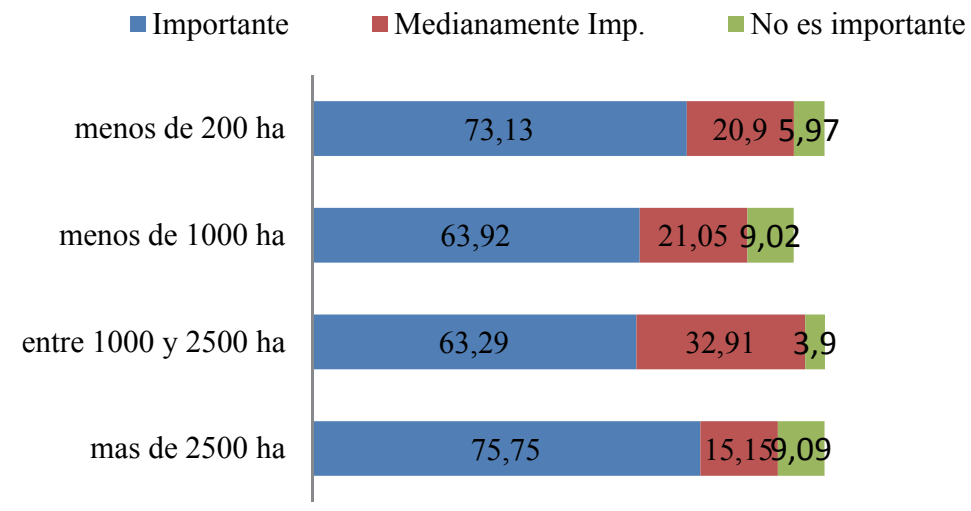

Fig. 4: porcentaje de opiniones afirmativas respecto a las variables de confiabilidad del producto de acuerdo a la importancia de la prestación del servicio técnico. 


\subsection{Variables sensibles de la percepción de la confiabilidad}

Las Variables Sensibles de la percepción de la confiabilidad de un producto agroindustrial según el orden de importancia son: "bajo índice de roturas imprevistas y desgastes prematuro" (variables agrupadas por su origen mecánico), "referencia de uso", "disponibilidad en tiempo"y "forma del servicio técnico por parte de fabricante". (Fig. 5).

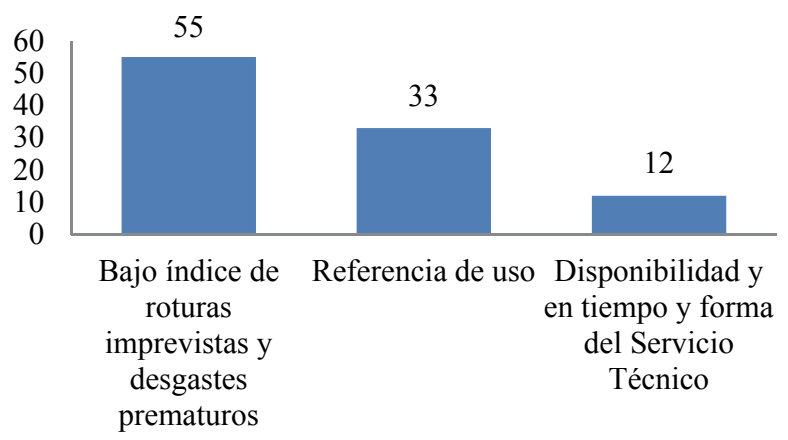

Fig. 5: Porcentaje de opiniones afirmativas respecto a las variables de confiabilidad del producto de acuerdo a la importancia de la prestación del servicio técnico.

De acuerdo a los resultados un producto se percibe como confiable (figura 5) cuando éste tiene en primer lugar bajo índice de "roturas imprevistas" y "desgastes prematuros" con un 55\%, en segundo lugar si existe "referencias de uso" en la zona más allá de conocer su performance operativa real. Es decir que el usuario conoce la existencia de máquinas similares en su zona pero no las prestaciones reales de la máquina por propio uso, variable importante como factor de decisión de compra.

El tercer factor: "disponibilidad en tiempo y forma del servicio tecnico" con $12 \%$, actuando ésta última como variable de equilibrio entre las dos primeras, esto se pone de manifiesto cuando los productores expresan "La máquina tiene algunos problemas de roturas y desgastes pero el servicio técnico es bueno por ese motivo la compré”.

\section{Discusión}

A vista de los resultados podemos decir que la percepción de confiabilidad de un producto agroindustrial como factor de compra está influenciada en primer lugar por la referencia del producto aceptando que mucha veces hay una distorsión en el mensaje. El productor no es un comprador que compra sin referencia, para el caso de las empresas que necesitan introducir su producto en una región territorial usualmente consignan una máquina a un productor radicado en el lugar para que la pruebe y la valore, en caso que esta cumpla con los objetivos de venta será la referencia para iniciar la actividad comercial. El conocimiento de la marca se ubica en el segundo lugar como factor de peso. Una marca puede ser conocida a través de una campaña publicitaria pero a la luz de la percepción de la confiabilidad como factor de compra, una es consecuencia de la otra. Las referencias positivas técnicas comerciales del vendedor/distribuidor en cierta medida se trasladan al producto que se comercializa esta asociación también se hace con el establecimiento fabril en el plano comercial, se evidencia en la variable información comercial, también sujeta desvíos respecto a la realidad.

El conocimiento de la fábrica puntualmente en el aspecto técnico, o sea como se fabrica el producto también es una variable que aporta a la percepción, pero muchas veces el productor desconoce los 
procesos de fabricación en detalle por lo que no relaciona la estabilidad de los mismo con la calidad final del producto, solamente aprecia la dimensión o la estética del mismo, de todas formas muchas pymes mediadas muestran sus procesos productivos ya sea a través de visitas guiadas o mediantes campañas publicitarias.

La percepción de la confiabilidad según el uso de la máquina va más allá de un concepto mecánico y económico, esta se construye y complementa primeramente cuando el producto no presenta "roturas imprevistas" ni "desgastes prematuros" . Esta variable se asocia al concepto mecánico de confiabilidad y a la noción dinámica, habiendo una relación entre los esfuerzos límites y a la duración de las piezas, teniendo como parámetro el tiempo otorgado como garantía por parte del fabricante.

La variable "servicio técnico" se puede considerar como una variable de equilibrio, explicando por qué máquinas de mayor calidad técnica pero con un servicio técnico que no satisface al cliente no tienen éxito en una determinada región. La variable facilidad de reparación también se puede considerar de equilibro, cuando el servicio técnico no es consecuente a la demanda.

Productores de menos del 200 ha en un $70 \%$ opinan que la variable servicio técnico es importante debido principalmente a las restricciones tecnológicas, no de tiempo. Por su parte, los productores de más de 2500 , también opinan que la variable servicio técnico es importante $(70 \%)$, en este caso debido principalmente a las restricciones de tiempo (datos no publicados).

Es evidente que las variables "roturas imprevistas" y " desgastes prematuros" se relacionan directamente con la gestión del diseño (Ortuño et al., 2000).También lo es la "facilidad de reparación" jugando un rol importante de equilibrio para aquellas empresas cuyo servicio técnico no satisface al potencial cliente por cuestiones logísticas y debe ser atendida como factor clave para la expansión empresarial.

\section{Conclusiones}

La percepción de confiabilidad de un producto agroindustrial como factor de compra está influenciada en primer lugar por la referencia del producto y por el conocimiento de la marca, existen otros que contribuyen en menor grado: referencia del vendedor/distribuidor, conocimiento de los procesos industriales del fabricante, información comercial. La percepción no se construye con una sola variable aislada.

La percepción de la confiabilidad según el uso de la máquina va más allá de un concepto mecánico y económico, esta se construye y complementa primeramente cuando el producto no presenta "roturas imprevistas" y " desgastes prematuros".

La variable "servicio técnico" se puede considerar como una variable de equilibrio.

Las variables "roturas imprevistas" y " desgastes prematuros", "facilidad de reparación" tienen una relación directa con la gestión del diseño.

\section{Bibliografía}

HERNANDIS, B.\& NAVARRO, E., (2000). Diseño de nuevos productos: una perspectiva sistémica. Universidad Politécnica de Valencia

SHIGLEY, J.,E BUDYNAS , R. y MISCHKE, C., (2004). Mechanical engineering design. McGraw-Hill.

BRAGACHINI, M (2010). Desarrollo industrial de ma maquinaria agrícola y agropartes en la Argentina. Impacto económico. 
$<\mathrm{http} / / /$ cosechaypostcosecha.org/data/articulos/maquinaria/DesarrolloIndustrialMaquinariAgricolaYAgropartes2011-02.pdf> [Consulta: 15 de Mayo de 2016]

SHKILIOVA, L.; CORONEL, C.; CABALLERO, A.(2007). Metodología para el cálculo de la productividad de las cosechadoras de arroz en función de la utilización del tiempo de turno. $<$ https://www.researchgate.net/profile/Alexander_Miranda2/publication/237042495_Metodologa_para_el_clculo_de la productividad de las cosechadoras de arroz en funcin de la utilizacin del tiempo de turno/links $/ 552429 \mathrm{da} 0$ cf2b123c517366 $\overline{\mathrm{d}}$.pdf $>$ [Consulta: 15 de Mayo de 2016].

SHKILIOVA, L. y FERNÁNDEZ SANCHEZ, M.(2011). Sistemas de Mantenimiento Técnico y Reparaciones y su aplicación en

la

Agricultura. $<$ http://scielo.sld.cu/scielo.php?script=sci arttext\&pid=S207100542011000100013\&lng=es\&nrm=iso $>$. ISSN 20710054.> [Consulta: 15 de Mayo de 2016]. 